\title{
Long timescale density peaking in JET
}

\author{
M Valovič ${ }^{1}$, J Rapp ${ }^{2}$, J G Cordey ${ }^{1}$, R Budny ${ }^{3}$, D C McDonald ${ }^{1}$, \\ L Garzotti ${ }^{4}$, A Kallenbach ${ }^{5}$, M A Mahdavi ${ }^{6}$, J Ongena ${ }^{7}$, V Parail ${ }^{1}$,

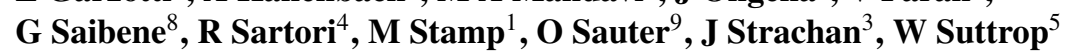 \\ and contributors to the EFDA-JET Workprogramme ${ }^{10}$
}

\author{
${ }^{1}$ EURATOM/UKAEA Fusion Association, Culham Science Centre, Abingdon, Oxfordshire \\ OX14 3DB, UK \\ ${ }^{2}$ Institut für Plasmaphysik, Forschungszentrum Jülich GmbH, Euratom Association, \\ D-52425 Jülich, Germany \\ ${ }^{3}$ PPPL, Princeton University, Princeton, NJ, USA \\ ${ }^{4}$ Consorzio RFX-Associazione Euratom-Enea sulla Fusione, Corso Stati Uniti 4, \\ I-35127 Padova, Italy \\ ${ }^{5}$ Max-Planck-Institut für Plasmaphysik, IPP-EURATOM Assoziation, Boltzmann-Str. 2, \\ D-85748 Garching, Germany \\ ${ }^{6}$ General Atomics, PO Box 85608, San Diego, CA 92138-9784, USA \\ ${ }^{7}$ LPP-ERM/KMS Association, Euratom-Belgian State, Brussels, Belgium \\ ${ }^{8}$ EFDA CSU Garching, Germany \\ ${ }^{9}$ CRPP Lausanne Centre de Recherches en Physique des Plasmas, Association
} EURATOM-Switzerland, EPFL, 1015 Lausanne, Switzerland

Received 16 January 2002

Published 27 August 2002

Online at stacks.iop.org/PPCF/44/1911

\begin{abstract}
Long timescale density peaking has been observed in JET plasmas leading to densities exceeding the Greenwald value. These neutral beam heated discharges are characterized by type-I ELMs and good energy confinement. Central density is limited by neoclassical tearing modes or by termination of $\mathrm{H}$-mode preceded by loss of sawteeth. When these limiting factors are avoided (i.e. at intermediate power level and with optimization of gas puffing) quasi-stationary high density plasmas with peaked density profile are obtained.
\end{abstract}

\section{Introduction}

Raising the core plasma density in tokamaks is the most effective way to maximize the fusion power for next-step devices. In ELMy H-mode, increasing the density by edge gas puffing generally results in a reduction of the energy confinement time to below the value predicted by the scaling law [1]. This confinement density limit has been attributed to the transition at the edge from type-I to type III ELMs. Recently it has been shown that the collapse of the plasma edge from type-I to type-III ELMy regime can be avoided if the gas puffing rate is restricted. In

${ }^{10}$ See annex of J Pamela 2001 et al Overview of recent JET results and future perspectives Fusion Energy 2000, Proc. 18th Int. Conf. (Sorrento, 2000) (Vienna: IAEA). 
such discharges with neutral beam heating the central density monotonically increases on a long timescale and exceeds the Greenwald density limit (GDL) while type-I ELMs and good energy confinement are preserved as observed in DIII-D [2,3], JET ${ }^{11}$ and ASDEX-Upgrade [4,5]. During this process the pedestal density is clamped close to the GDL leading to a peaked density profile. Note that pedestal densities at the GDL have recently been achieved in JET [6]. This paper reports on the scenario with density peaking on JET and identification of limiting factors.

\section{Type-I ELMy H-mode above Greenwald density limit}

A key ingredient of these experiments are the use of moderate gas fuelling rates. It is known that there is a limit for gas fuelling rate above which the transition from type-I to type-III ELM regime occurs leading to a degradation of edge transport barrier and deterioration of particle and energy confinement. This threshold increases with triangularity [1]. Its dependence on heating power is not yet systematically established. However, it has been demonstrated recently [6, 7], that this limit exists also at higher heating powers and plasma currents then originally reported [1]. Second condition for achieving these regimes is that the duration of the discharges has to be extended to allow for long timescale evolution of the density profile. Initial experiments were with low field $\left(B_{\mathrm{T}}=1 \mathrm{~T}, I_{\mathrm{p}}=0.95 \mathrm{MA}\right)$ and low triangularity shape $(\delta=0.26)$. With fuelling from outboard midplane of the main chamber at the rate of $2 \times 10^{21} \mathrm{el} \mathrm{s}^{-1}$ and heating power $P_{\mathrm{NBI}}=3 \mathrm{MW}$ the line-averaged density was linearly increasing for $9 \mathrm{~s}$ and reached $\bar{n} / n_{\mathrm{Grw}} \approx 1.1$ (pulse no $\left.50650, n_{\mathrm{Grw}}\left(10^{20} \mathrm{~m}^{-3}\right)=I_{\mathrm{p}}(\mathrm{MA}) / \pi a^{2}\right)$. Low frequency $(4 \mathrm{~Hz})$ type-I ELMs were preserved. The density at the top of the pedestal $(r / a \approx 0.9)$ is clamped at $n_{\text {ped }} \approx 0.85 n_{\text {Grw }}$ leading to a peaked density profile with central density of $n_{0} \approx 1.4 n_{\text {ped }}$. Similar results have been obtained at a medium triangularity $\left(\delta_{\mathrm{L}}=0.27, \delta_{\mathrm{U}}=0.34\right)$, higher field $B_{\mathrm{T}}=1.7 \mathrm{~T}, I_{\mathrm{p}}=1.6 \mathrm{MA}$ and fuelling from outer divertor ring at the rate of $2 \times 10^{22} \mathrm{el} \mathrm{s}^{-1}$. At $P_{\mathrm{NBI}}=5.7 \mathrm{MW}$ densities of $\bar{n} / n_{\mathrm{Grw}} \approx 1.1$ have been reached with the timescale of $2-4 \mathrm{~s}$ (pulse no 52458).

Most of the studies, however, have been done at high triangularity $\left(\delta_{\mathrm{L}}=0.4, \delta_{\mathrm{U}}=0.6\right)$ and higher field $\left(B_{\mathrm{T}}=2 \mathrm{~T}, I_{\mathrm{p}}=1.9 \mathrm{MA}\right)$. The gas was supplied from the outer divertor ring. An example of such a plasma is shown in figure 1 . The line-averaged density monotonically increases with a timescale of several seconds and exceeds the GDL by up to $\sim 12 \%$. The pedestal density is limited to $\sim 80 \%$ of the GDL leading to a ratio of core to pedestal density of $\sim 1.5$. During this process type-I ELMs and good energy confinement are preserved. With the same shape this scenario has been reproduced even at higher field $\left(B_{\mathrm{T}}=2.7 \mathrm{~T}, I_{\mathrm{p}}=2.5 \mathrm{MA}\right)$ and power $\left(P_{\mathrm{NBI}}=13.5 \mathrm{MW}\right)$. In this case (pulse no 52015) the gas puffing is from inner divertor ring at the rate of $1.5 \times 10^{22} \mathrm{el} \mathrm{s}^{-1}$. The line-averaged density was increasing for $6 \mathrm{~s}$ reaching $\bar{n} / n_{\text {Grw }} \approx 1.0$ with highly peaked density profile $n_{0} \approx 1.5 n_{\text {ped }}$ while preserving low frequency $(19 \mathrm{~Hz})$ type-I ELMs.

\section{NBI power scan}

The response of maximum achievable density on heating power was investigated at the same plasma current, field, shape and gas puffing rate as in figure 1. The results are summarized in figure 2 showing the central density as a function of central electron temperature.

For NBI power below $8 \mathrm{MW}$ the central electron temperature is lower than $\sim 2 \mathrm{keV}$ (figure 2). These plasmas are transient. Central density increases linearly in time.

${ }^{11}$ Long timescale density peaking has been also retrospectively identified in some earlier JET discharges, e.g. shot no 48276. 

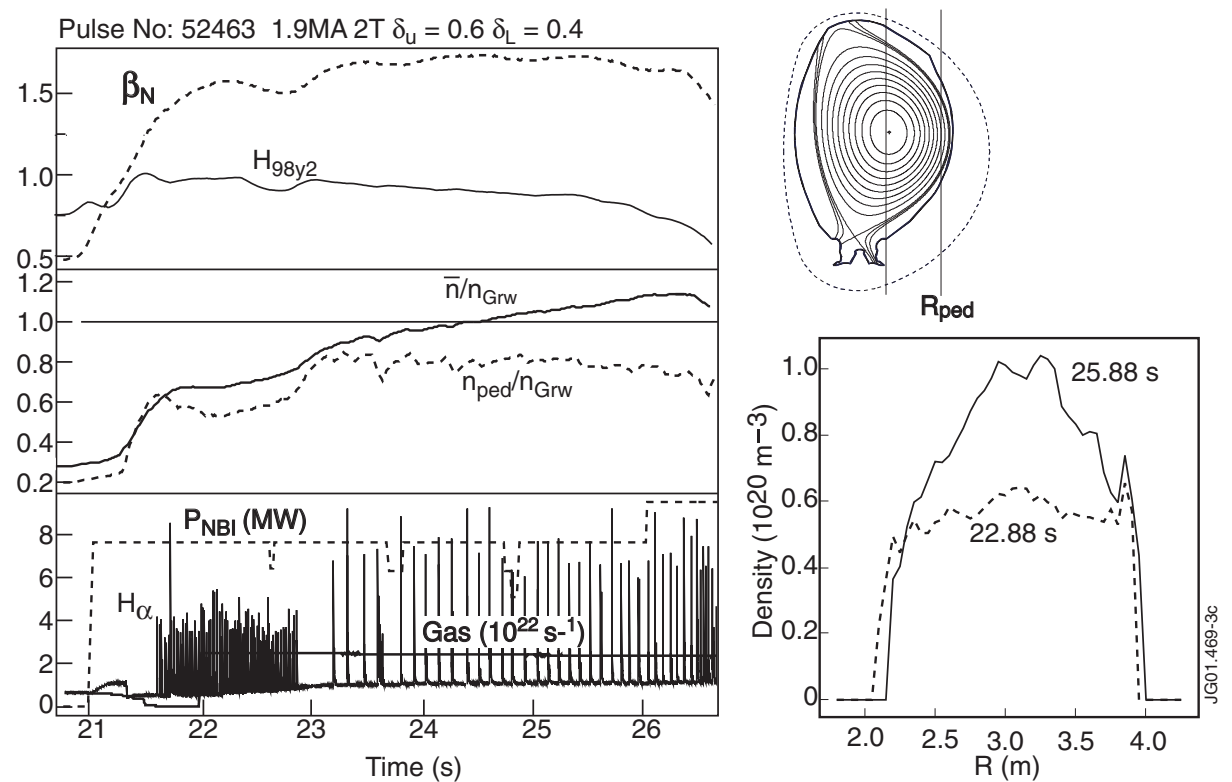

Figure 1. Discharge above the Greenwald density limit with type-I ELMs.

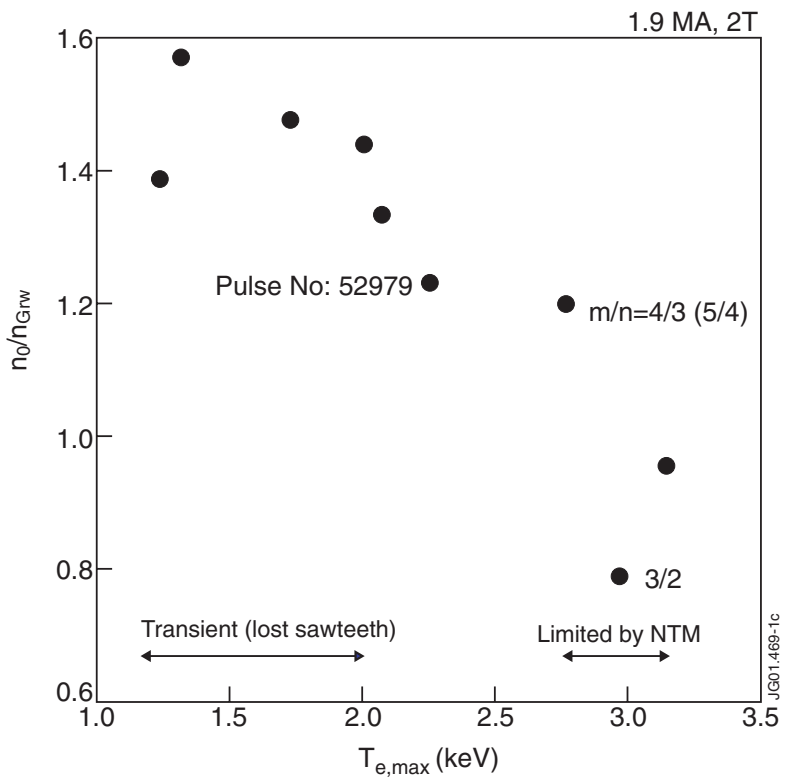

Figure 2. Dependence of central density on central electron temperature in power scans.

Simultaneously the current density profile is broadening as indicated from the decrease of internal inductance and the rise of central safety factor and eventually sawteeth disappear. The loss of sawteeth results in an even larger increase of core density and at its maximum exceeding the GDL by $30-40 \%$. The increase of the core density results in further reduction of core temperature and the $\mathrm{H}$-mode is terminated by enhanced core radiation. In these experiments, 
the periods without sawteeth were short and gradual increase of core density is stopped by $\mathrm{H}-\mathrm{L}$ transition. There is, however, a case where linearly growing mode terminates the density peaking after sawteeth have disappeared. This could be related to the ASDEX-Upgrade observation [5], were early onset of neoclassical tearing mode (NTM) can occur without sawtooth trigger as a result peaked density profile and consequent enhancement of bootstrap current. However, the onset of mode as a function of the density gradient and contribution from different driving terms in tearing mode equation were not studied systematically.

At higher NBI power, $12-14 \mathrm{MW}$, the central electron temperature is above $\sim 2.5 \mathrm{keV}$ (figure 2) and the temperature collapse is avoided. These plasmas preserve regular sawteeth activity throughout the duration of the heating pulse. At these power levels $n_{0}$ is limited by NTMs. Typically modes with high mode numbers $m / n=4 / 3(5 / 4)$ occur first. Already these modes are observed to stop the monotonic increase of $n_{0}$ resulting in quasi-stationary plasma. When large $m / n=3 / 2$ modes occur $(7-10 \mathrm{~cm})$ the core density drops to the pedestal value leading to flat density profile. It is known that NTMs could be triggered by sawteeth. No attempts has been made to reduce the size of sawteeth in this scan for example by ion cyclotron current drive, the technique that has recently been successfully demonstrated [8]. Another trigger for NTMs observed in this scan was the large ELM usually at the beginning of type-I ELMy phase of the discharge. Moderation of the ELM size was the key for achieving the quasi-stationary discharge with peaked density profile described below. The occurrence of NTMs in this power range does not allow us to clearly separate how much the flattening of density profile is caused by changes of underlying particle transport due to the increased core temperature and how much due to the NTMs. Nevertheless, the onset of NTMs could be one of the practical limits on density peaking in fusion devices.

There is a narrow window in NBI heating power (around $\sim 10 \mathrm{MW}$ ) where sawteeth are preserved and simultaneously large NTMs can be avoided resulting in a quasi-stationary high density plasmas. An example of such discharge is shown in figure 3. This plasma is also a result of further optimization of gas puff. It uses fuelling from the inner ring on the divertor

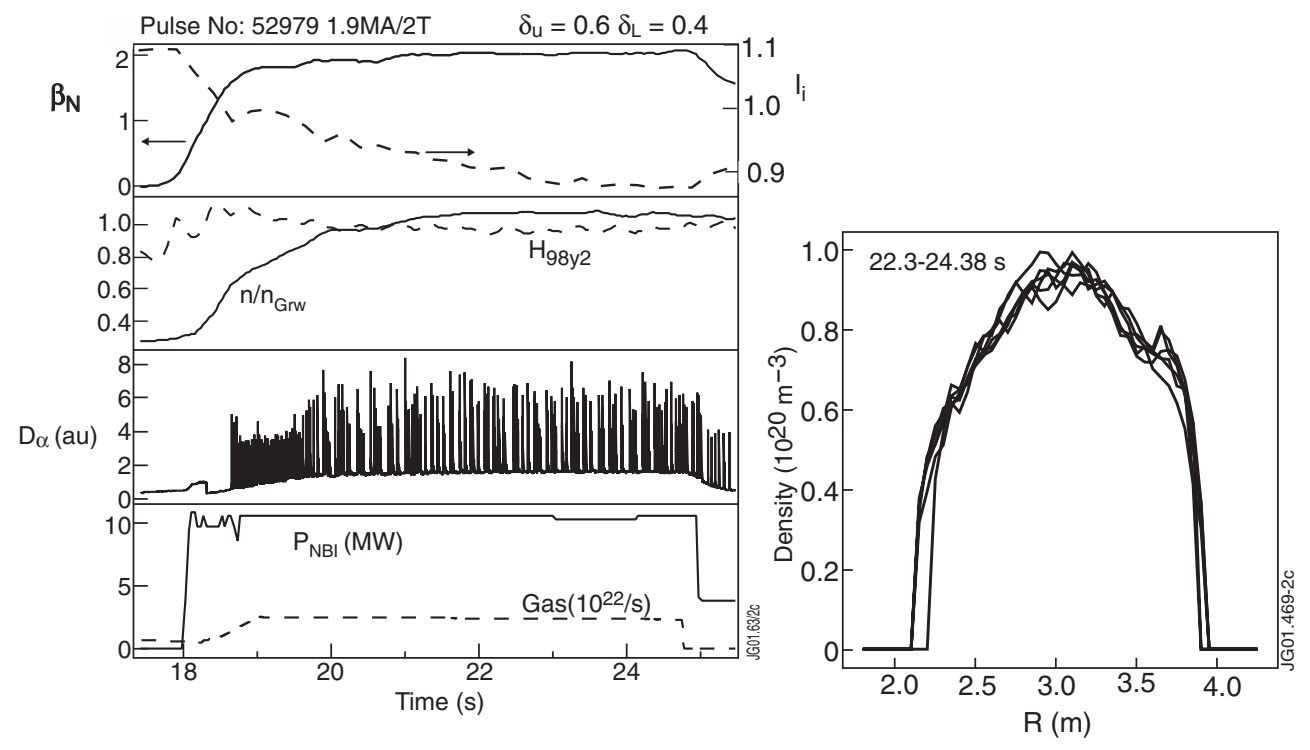

Figure 3. Quasi-stationary ELMy H-mode plasma at the Greenwald density limit. 
base (puffing into the private region) as this has been found to be the optimum from gas puff position scan. Simultaneously the gas waveform has been optimized in order to moderate the size of the first ELM after the ELM-free phase which usually triggers a large $m / n=3 / 2$ NTM. In the shot in figure 3 the sawteeth are preserved to the end of the heating phase. The decrease of internal inductance is still evident but this trend slows towards the end of heating phase. The density profile reaches equilibrium leading to a quasi-stationary discharge with line averaged density $\bar{n} / n_{\mathrm{Grw}} \approx 1.0$. The central density is about $30 \%$ higher than the pedestal density. This density peaking is weaker in comparison with the extremely peaked profiles obtained in transient discharges described above. The discharge in figure 3 exhibits good normalized energy confinement time of $H_{\mathrm{H} 98 \mathrm{y} 2}=0.96$ at $\beta_{\mathrm{N}}=2$, and thus, contributes to the confinement database in the vicinity of ITER operational point [9].

As mentioned above, in cases with density peaking, the plasma current profile broadens and central safety factor $q(0)$ increases. In both transient (figure 1) and quasi-stationary case (figure 3 ) the effective charge increases only slightly during the sawtoothing phase of the discharges $\left(Z_{\text {eff }} \sim 1.5-1.6\right.$ from chord average brehmstrahlung radiation). Therefore the raise of $q(0)$ seems to be a consequence of gradual decrease of central electron temperature rather than impurity accumulation. However, after the loss of sawteeth (at $t>25.8 \mathrm{~s}$ in figure 1) increase of central $Z_{\text {eff }}$ is observed by charge exchange resonance spectroscopy.

\section{Ion cyclotron heating}

In the power scan (figure 2) the reduced peaking at higher temperatures was found to correlate with the onset of NTMs. It has been suggested, on the basis of ASDEX-U data [4,5] that increasing the temperature gradient results in an increase of turbulent transport and this leads to a flat density profile. On the other hand, theory predicts that turbulence itself can generate particle pinch [10]. In order to address this issue experiments with ICR heating were performed (second harmonic, $f=51.2 \mathrm{MHz}, \mathrm{H}$-minority, $B_{\mathrm{T}}=1.7 \mathrm{~T}, I_{\mathrm{p}}=1.6 \mathrm{MA}$ ). NBI was still used but it contributed in $25 \%$ of the total heating power. In these plasmas even at $8 \mathrm{MW}$ of total power, the central electron temperature stays at $\sim 2 \mathrm{keV}$ and sawteeth are preserved. The core density is high, $n_{0} / n_{\text {Grw }} \approx 1.1$, and the density profiles rather flat (high pedestal density). Global energy confinement is relatively low in these high density ICRH plasmas $\left(H_{\mathrm{H} 98 \mathrm{y} 2}=0.6\right)$. These discharges have much higher ELM frequency in comparison with NBI heated plasmas indicating perhaps different edge conditions due to RF coupling.

\section{Transport analysis}

Analysis was done for the quasi-stationary plasma shown in figure 3 using the JETTO code. At $t=23 \mathrm{~s}, \mathrm{~d} n / \mathrm{d} t=0$, so that the particle ion flux is outwards and equal to the NBI source. Inside the $\rho=0.4$ surface this source is $\Phi=2.6 \times 10^{20} \mathrm{~s}^{-1}$, which gives the flux density $\Gamma=\Phi / \Sigma=0.063 \mathrm{~m} \mathrm{~s}^{-1} \times n_{\mathrm{i}}$. At the same radius the Ware pinch velocity is $V_{\mathrm{W}} \approx 0.04 \mathrm{~m} \mathrm{~s}^{-1}$ (figure 4). This value is comparable to $\Gamma / n_{\mathrm{i}}$ and therefore the pinch and NBI fuelling play an equal role in density peaking.

In quasi-stationary conditions, as in figure 3, convective and diffusive particle fluxes cannot be distinguished. The pinch term could be estimated from impurity puff experiments (e.g. helium); however, these were not performed for these shots. If only neoclassical pinch velocity $V=V_{\mathrm{W}}$ is assumed then the ion diffusion coefficient at $\rho=0.4$ is $D=\left(\Gamma / n_{\mathrm{i}}+\left|V_{\mathrm{W}}\right|\right) \times L_{\mathrm{n}}=0.28 \mathrm{~m}^{2} \mathrm{~s}^{-1}$, where $L_{\mathrm{n}}=n_{\mathrm{i}} / \nabla n_{\mathrm{i}}=2.4 \mathrm{~m}$ is the density scale length. At the same radius, the effective heat conductivity is $\chi_{\mathrm{eff}}=1.2 \mathrm{~m}^{2} \mathrm{~s}^{-1}$ so that the ratio 


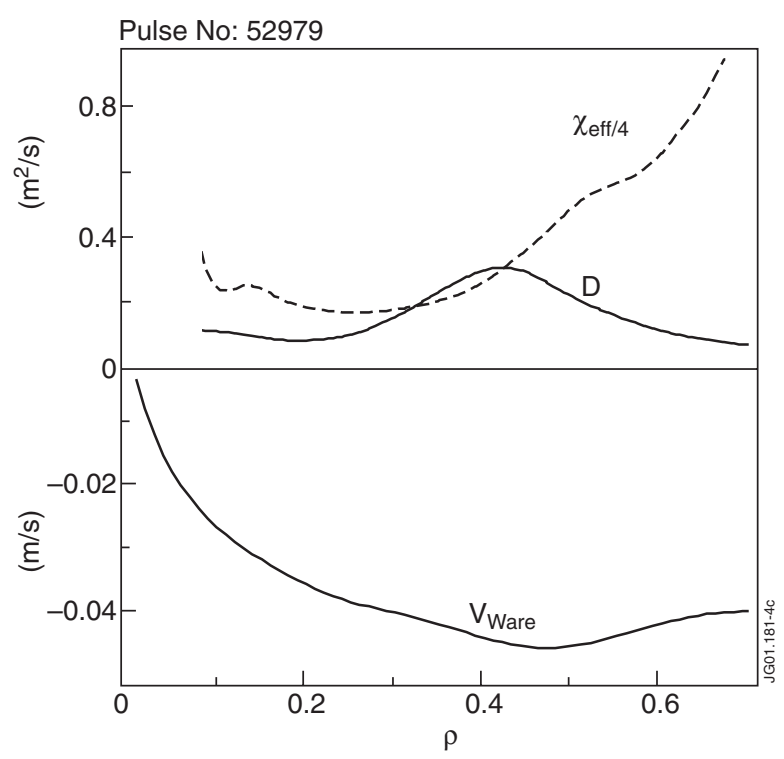

Figure 4. Ion particle diffusion coefficient $D$, effective thermal diffusivity $\chi_{\text {eff }}$ and Ware pinch velocity $V_{\mathrm{W}}$ for pulse no 52979 at time $22.8 \mathrm{~s}$ (JETTO).

$D / \chi_{\text {eff }} \sim 0.25$ (see figure 4). Assuming a strong anomalous pinch, say $V=10 V_{\mathrm{W}}$, results in particle diffusion coefficient of $D / \chi_{\text {eff }} \sim 1$. In this case, $V \gg \Gamma / n_{\mathrm{i}}$, and the pinch would control the density peaking.

It is outside the scope of this paper to determine which values of $D / \chi_{\text {eff }}$ are compatible with theory. The value of $D / \chi_{\text {eff }} \sim 1$ is rather large and perhaps indicates that $V=10 V_{\mathrm{W}}$ is the upper limit for anomalous pinch at quasi-stationary sawtoothing conditions. Comparison of numerical values of measured transport coefficients with theory is more complicated if off-diagonal elements of transport matrix are considered. Strong density peaking that occurs after the loss of sawteeth in the transient case in figure 1 is well reproduced with the Weiland transport model which includes off-diagonal elements [11].

One source of uncertainty is the error in the particle source. TRANSP analysis, using Monte Carlo method for particle deposition, for the same shot and time as in figure 4 shows less beam deposition in the core than calculated by JETTO using. Consequently the values for particle and heat diffusivites in the core are lower. Assuming $V=V_{\mathrm{W}}$ the ratio of particle and heat difussivity calculated by TRANSP at $\rho=0.4$ is $D / \chi_{\text {eff }} \sim 0.4$. This value is somewhat higher than that calculated by the JETTO code. This difference is larger than the error in ion diffusivity due to the uncertainty in the density scale length at $\rho \sim 0.4(\sim 25 \%)$.

As already discussed, in ICRH dominated shots, the density profiles were not peaked. However, the conditions at the edge (ELMs, recycling) were different from those in NBI plasmas and more study is required for particle transport in ICRH plasma.

\section{Conclusions}

With reduced gas puffing and allowing for longer timescales, plasmas with central densities up to $1.4 n_{\mathrm{Grw}}$ can be transiently achieved with low frequency type-I ELMs. At higher NBI heating power the density peaking is limited by NTMs. With gas puff optimization and intermediate heating power a quasi-stationary discharge with $\bar{n} / n_{\mathrm{Grw}}=1.0, H_{\mathrm{H} 98 \mathrm{y} 2}=0.96, \beta_{\mathrm{N}}=2$ and 
density peaking of $n_{0} / n_{\text {ped }}=1.3$ is obtained. These plasmas contribute to improvements of energy confinement scaling at high densities.

\section{Acknowledgment}

This work has been conducted under the European Fusion Development Agreement and is partly funded by Euratom and UK Department of Trade of Industry.

\section{References}

[1] Saibene G et al 1999 Nucl. Fusion 391133

[2] Osborne T H et al 2001 J. Nucl. Mater. 290-293 1013

[3] Mahdavi M A et al 2000 18th IAEA Conf. (Sorrento, 2000) IAEA-CN-77EXP1/04

[4] Stober J et al 2001 Nucl. Fusion 411535

[5] Stober J et al 2001 Plasma Phys. Control. Fusion 43 A39

[6] Saibene G et al 2002 Plasma Phys. Control. Fusion 441769

[7] Sartori R et al 2002 Plasma Phys. Control. Fusion 441801

[8] Sauter O et al 2002 Phys. Rev. Lett. 88 105001-1

[9] Cordey J G et al 2002 Plasma Phys. Control. Fusion 441929

[10] Weiland J 2001 Proc. 28th EPS Conf. on Controlled Fusion and Plasma Physics (Funchal, 2001) Europhys. Conf. Abstracts 25A 633

[11] Parail V V 2001 Proc. 28th EPS Conf. on Controlled Fusion and Plasma Physics (Funchal, 2001) Europhys. Conf. Abstracts 25A 1873 